\title{
Comparative Study of Mandible Ramus Morphology Using 3-dimensional CT in Sagittal Split Ramus Osteotomy
}

\author{
Masayuki Hirota $^{1)}$, Kenichirou Suga ${ }^{2)}$ and Takahiko Shibahara ${ }^{1)}$ \\ ${ }^{1)}$ Department of Oral and Maxillofacial Surgery, Tokyo Dental College, \\ 2-9-18 Kanda-Misakicho, Chiyoda-ku, Tokyo 101-0061, Japan \\ 2) Department of Dentistry, Oral Surgery and Pediatric Dentistry, Tochigi Medical Center, \\ 1-10-37 Tomatsuri, Utsunomiya-shi, Tochigi 320-8580, Japan
}

Received 24 December, 2017/Accepted for Publication 16 January, 2018

Published Online in J-STAGE 18 October, 2018

\begin{abstract}
The purpose of the present study was to investigate the relevance of the external morphology of the mandibular ramus, internal bone tissue structure, and maxillofacial morphology at the site for sagittal split ramus osteotomy among different facial skeletal patterns. A total of 80 patients with jaw deformities who underwent sagittal split ramus osteotomy were included in the study. The patients were divided into two groups based on facial skeletal type (skeletal Class II or skeletal Class III). A further 7 patients with no skeletal abnormalities were established as the control group (skeletal Class I). Computed tomography images obtained from these patients were 3-dimensionally reconstructed and the morphology of the mandibular ramus determined. Thickness at the mandibular foramen in Class II was greater than that in Class III, and showed the lowest value at the midpoint of the mandibular foramen and mandibular notch in Class I. Mandibular morphology showed change according to facial skeletal type. Correlations were also observed between the cephalometric analysis values and mandibular morphology.
\end{abstract}

Key words: Bone morphometry - Computed tomography -

Mandible ramus - Osteotomy — Oral and maxillofacial surgery

\section{Introduction}

Introduced in 1957, sagittal split ramus osteotomy is used to correct deformities of the mandible ${ }^{20)}$. This technique has become established as a standard procedure because it offers many advantages. Its goal is to reposition the mandibular body by splitting the mandibular ramus and body into two layers buccolingually. This leaves a large surface area of bone contact after bone fragment movement, which is effective in preventing

This paper was originally presented as a thesis by M. Hirota at the Graduate School of Tokyo Dental College. 
regression and curtailing intermaxillary fixation time $\mathrm{e}^{1,5,7,18)}$. It is also advantageous in that there is a high degree of freedom with respect to the direction of movement of the mandibular body, and in that it avoids the mandibular canal. The mandibular ramus thins buccolingually, however, and the mandible itself is a complex structure which contains the mandibular canal. This means that special care must be taken to avoid damaging the mandibular canal or ectopic bone when performing bone splitting. Moreover, the surgical field is deep and narrow, so serious accidents, such as airway constriction due to swelling or bleeding, can easily occur if the mandibular canal is damaged. Therefore, to avoid this, various proposals have been made for procedures that improve the position or length of the osteotomy ${ }^{2,14,17}$. A procedure that would be suitable for all types of maxillofacial deformity remains to be found, however. Furthermore, criteria for selecting the osteotomy line regardless of disease type remain to be established.

A number of studies have investigated the anatomy of the mandible using specimens obtained from cadavers ${ }^{13,16)}$. There have also been studies examining the positional relationship with buccal cortical bone in jaw deformities using computed tomography (CT) images, as well as studies investigating the positional relationship with the bone marrow $^{3,8,23)}$. Classifications for the mandibular canal course have also been reported ${ }^{13)}$. To our knowledge, however, no studies to date have comprehensively assessed the external morphology of the mandible, the position and buccolingual thickness of the mandibular foramen, or the course of the mandibular canal; and, moreover, none has comparatively investigated patterns of facial deformity or differences in mandibular ramus morphology and internal structure.

The purpose of the present study, therefore, was to quantitatively observe the mandibular ramus and body in relation to the site for sagittal split ramus osteotomy among different facial skeletal types (Class I to III), using a 3-dimensional (3D) CT-image pro- cessing system. The relevance of maxillofacial morphology, the external morphology of the mandibular ramus, and internal bone tissue structure was also investigated.

\section{Materials and Methods}

The study participants comprised 80 patients (mean age, 25.6 years, ranging 21 to 37 years; 25 men and 55 women) with jaw deformities visiting the Department of Oral \& Maxillofacial Surgery at the Tokyo Dental College Chiba Hospital over the 7 years spanning April 2007 to March 2013, where all underwent surgical orthodontic treatment. All were free of congenital disease and syndromes. Patients with missing molars or facial asymmetry (where the midline of the mandibular dentition showed a left-right deviation of $3 \mathrm{~mm}$ or more) were excluded. All eligible for inclusion were classified as skeletal Class II or Class III in accordance with the method of Naruse $^{12}$. A further 7 patients with malocclusion but no skeletal abnormalities, and who were classified as Class I based on CT images, comprised the control group. Table 1 shows the age and sex for each skeletal type and the control group.

Cephalometric analysis values and CT images were obtained after completion of preoperative correction in the Class II and Class III groups prior to performance of sagittal split ramus osteotomy; the same were obtained in the Class I patients prior to corrective implant anchor placement. Six items pertaining to skeletal pattern and 2 to overjet and overbite were measured for the cephalometric analysis, making a total of 8 altogether, according to the Downs and Northwestern methods. A Siemens Somatom Plus 4 Volume Zoom (Siemens Japan, Tokyo, Japan) was used for the CT. Continuous slice images were obtained under the following conditions: tube voltage, $120 \mathrm{kVp}$; tube current, $100 \mathrm{~mA}$; reconstruction slice thickness, $1.25 \mathrm{~mm}$; reconstruction slice interval, $1.0 \mathrm{~mm}$; scan speed, $0.75 \mathrm{sec} /$ rotation; and movement speed, $3 \mathrm{~mm} / \mathrm{sec}$. All image data were output- 
Table 1 Patients character

\begin{tabular}{lrcc}
\hline \hline & \multicolumn{1}{c}{ Class I } & Class II & Class III \\
\hline Total & 7 & 33 & 47 \\
Sex & & & \\
$\quad$ Male & 0 & 7 & 18 \\
Female & 7 & \multicolumn{1}{c}{26} & \multicolumn{1}{c}{29} \\
Age (Mean \pm SD) & $21.6 \pm 2.9$ & $26.9 \pm 2.5$ & $26.3 \pm 3.1$ \\
\hline Cephalometric analysis & & & \\
\hline Facial angle & $85.7 \pm 3.6$ & $82.3 \pm 3.2$ & $96.7 \pm 2.1$ \\
Y-axis & $68.3 \pm 3.3$ & $70.6 \pm 4.8$ & $62.7 \pm 4.3$ \\
SNA & $80.6 \pm 3.7$ & $79.5 \pm 3.2$ & $77.3 \pm 4.3$ \\
SNB & $76.7 \pm 4.8$ & $73.8 \pm 3.1$ & $80.5 \pm 3.6$ \\
ANB & $3.7 \pm 2.1$ & $5.3 \pm 1.2$ & $-3.4 \pm 1.3$ \\
Gonial angle & $125.2 \pm 4.7$ & $119.6 \pm 3.1$ & $129.3 \pm 5.7$ \\
overjet (mm) & $2.3 \pm 0.9$ & $3.2 \pm 1.2$ & $-2.9 \pm 1.5$ \\
overbite (mm) & $2.3 \pm 0.7$ & $3.0 \pm 1.2$ & $1.0 \pm 1.3$ \\
\hline
\end{tabular}

ted in Digital Imaging and Communications in Medicine (DICOM) format. Measurements were taken from the reconstructed mandibles obtained using the slice image 3D conversion software Mimics (Materialise Japan, Yoko hama, Japan). The threshold for cortical bone area setting was set at $50 \%$ of the CT value difference.

Written informed consent was obtained from all study participants and the study protocol approved by the Ethics Committee of Tokyo Dental College (Clearance Number, 556).

\section{Reference points used for 3D constructed CT}

The obtained CT images were 3D reconstructed on a personal computer. Six items were set for reconstruction of the mandible according to the method of Tsuji ${ }^{21)}$. In addition, 6 further items ([5] through [10]) were newly added to express the position of the mandibular foramen and course of the mandibular canal in detail (Figs. 1, 2). That is, they were divided into the mandibular ramus and body, and the 12 reference points set. The occlusal plane was used as the reference, and was set using the distal buccal cusp of the first molars and the midpoint of the mesial corner of the mandible central incisor.

\section{Measurement items}

From the reference points used for 3D reconstructed CT, measurements were taken for a total of 10 items. Values were calculated using the mean of the bilateral measurements as an individual value ( $\mathrm{mm}$ ) (Table 2).

\section{Statistical analysis}

All statistical analyses were performed using the statistical analysis program SPSS. The Mann-Whitney test was used for intergroup comparisons among the 3 skeletal facial types.

Multiple regression analysis was performed for each class using each measurement value from the 3D CT images as a dependent variable and facial angle, Y-axis, SNA, SNB, angle between the maxilla and the mandible (ANB), gonial angle, overjet, and overbite as independent variables. 


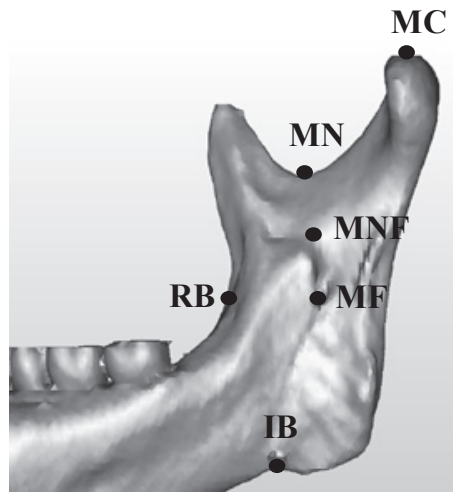

Fig. 1 Reference points in mandibular ramus MF: mandibular foramen [1], RB: mandibular ramus anterior border [2], MC: mandibular condyle [3], IB: mandibular inferior border [4], MN: mandibular notch [5], MNF: midpoint of mandibular notch and mandibular foramen [6].

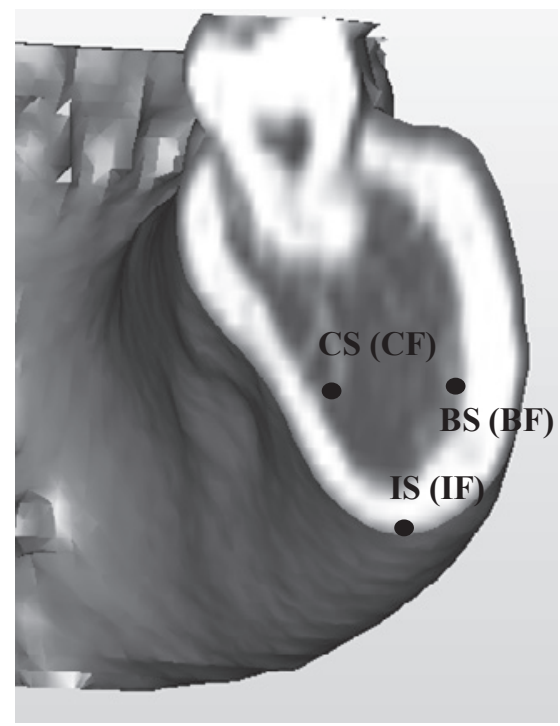

Fig. 2 Reference points on mandibular body

IF: position of mandibular inferior border at first molar [7], IS: position of mandibular inferior border at second molar [8], CF: position of mandibular canal at first molar [9], CS: position of mandibular canal at second molar [10], BF: position of buccal cortical bone at first molar [11], BS: position of buccal cortical bone at second molar [12].

Table 2 Measurement items for the mandibular ramus and body

T-MF Thickness at the mandibular foramen. Buccolingual thickness dimension of MF

T-MNF Measuring the buccolingual thickness dimension of MNF at the midpoint of the mandibular notch and the mandibular foramen

MN-MF Distance of the mandibular foramen from the mandibular notch using MF and MN

IB-MF Distance of the mandibular foramen from the mandibular inferior border using IB and MF

RB-MF Distance of the mandibular foramen from the mandibular ramus anterior border using RB and MF

MC-IB Measuring the height dimension of the mandibular ramus using MC and IB

IF-CF Distance of the mandibular canal from the mandibular inferior border at the position of the first molar using IF and CF

BF-CF Distance of the mandibular canal from the buccal cortical bone at the position of the first molar using $\mathrm{BF}$ and $\mathrm{CF}$

IS-CS Distance of the mandibular canal from the mandibular inferior border at the position of the second molar using IS and CS

BS-CS Distance of the mandibular canal from the buccal cortical bone at the position of the second molar using BS and CS 


\section{Results}

\section{Measurement values for 3D CT images}

With respect to the mandibular ramus, Class II had a larger T-MF $(13.9 \pm 1.4 \mathrm{~mm}$, mean $\pm \mathrm{SD})$, an item representative of thickness, than Class III $(12.7 \pm 1.4 \mathrm{~mm})(\mathrm{p}<0.01)$; and T-MNF was smallest for Class I $(12.7 \pm 0.9 \mathrm{~mm})$, followed by Class II $(11.0 \pm 1.8 \mathrm{~mm})$ and Class III $(8.5 \pm 1.3 \mathrm{~mm})$ $(\mathrm{p}<0.01)$. Class II $(22.3 \pm 2.2 \mathrm{~mm})$ and Class III $(21.9 \pm 2.3 \mathrm{~mm})$ had a smaller MN-MF, an item representative of the position of the mandibular foramen, than Class I $(25.6 \pm 1.2 \mathrm{~mm}) \quad(\mathrm{p}<0.05)$; and Class III $(25 \pm 2.1 \mathrm{~mm})$ had a greater IB-MF than Class I $(27.1 \pm 1.1 \mathrm{~mm})$ or Class II $(26.5 \pm 1.9 \mathrm{~mm})$ $(\mathrm{p}<0.05)$. The RB-MF and MC-IB showed no significant differences among the facial skeletal types.

With respect to the mandibular body, Class II had a larger IF-CF $(15.1 \pm 1.3 \mathrm{~mm})$, an item representative of the distance from the mandibular inferior border, than Class III $(16.0 \pm 1.8 \mathrm{~mm})(\mathrm{p}<0.05)$; and Class II had a smaller IS-CS $(17.2 \pm 1.5 \mathrm{~mm})$ than Class III $(17.4 \pm 2.2 \mathrm{~mm}) \quad(\mathrm{p}<0.05)$. Class II had a smaller BS-CS $(2.6 \pm 1.4 \mathrm{~mm})$, an item representative of the distance of the mandibular canal from the buccal cortical bone, than Class III $(3.4 \pm 1.2 \mathrm{~mm})(\mathrm{p}<0.05)$ (Table 3$)$.

\section{Correlations between results of cephalometric analysis and measurement values}

In Class I, a negative correlation was observed between SNB and MC-IB ( $p=0.048)$, a measurement item representative of the mandibular ramus. No correlation was observed between other items representative of the mandibular ramus or items representative of the mandibular body.

In Class II, IB-MF, an item representative of the mandibular ramus, showed a negative correlation with overjet $(p=0.026)$. The RB-MF showed positive correlations with SNA $(p=0.033)$ and overjet $(p=0.044)$. The MC-IB showed a particularly strong negative correlation with overjet $(p=0.007)$. No cor- relation was observed between other items representative of the mandibular ramus or items representative of the mandibular body.

In Class III, T-MNF, an item representative of the mandibular ramus, showed a positive correlation with SNB $(\mathrm{p}=0.011)$, as did RB-MF with overbite $(p=0.017)$ and MC-IB with the Y-axis $(p=0.017)$. The MN-MF showed a negative correlation with facial angle $(p=0.023)$, as did MC-IB with overbite $(p=0.048)$. No correlation was observed with other items representative of the mandibular ramus. The BF-CF, an item representative of the mandibular body, showed a positive correlation with the $\mathrm{Y}$-axis $(\mathrm{p}=0.002)$ and facial angle $(\mathrm{p}=0.005)$, as did BS-CS with the Y-axis $(p=0.004)$ and facial angle $(p=0.004)$. These values showed a strong correlation with this item of the mandibular body. No correlation was observed with other items representative of the mandibular body, however.

In Class II, correlations were observed between items from the cephalometric analysis and items pertaining to the mandibular ramus on 3D CT images; no correlations were observed with items pertaining to the mandibular body, however. In Class III, the cephalometric analysis values showed correlations with both items representative of the mandibular ramus and those representative of the mandibular body on 3D CT images (Table 4).

\section{Discussion}

Approximately 650 surgical procedures are performed under general anesthesia at our hospital each year, and about 120 of these $(18 \%)$ are sagittal split ramus osteotomies. This technique sometimes results in nerve damage, abnormal bone fracture, or other adverse events ${ }^{11,22)}$. These accidents are thought to be associated with skeletal abnormalities. Currently, sagittal split ramus osteotomy is preceded by CT image examination and diagnosis of mandibular morphology $y^{15,19)}$. In recent years, CT imaging has been widely used in dental treatment to obtain useful preoperative information when a sagittal split 
Table 3 Anatomic analysis

\begin{tabular}{|c|c|c|c|c|c|}
\hline \multirow{2}{*}{ Measurement (mm) } & \multicolumn{2}{|l|}{ Class I $(\mathrm{n}=7)$} & \multicolumn{2}{|l|}{ Class II $(\mathrm{n}=33)$} & \multirow{2}{*}{$\begin{array}{c}\text { Class III }(\mathrm{n}=47) \\
\text { Mean } \pm \mathrm{SD}\end{array}$} \\
\hline & Mean \pm SD & & Mean \pm SD & & \\
\hline \multicolumn{6}{|l|}{ Mandibular ramus } \\
\hline \multirow[t]{2}{*}{ T-MF } & \multicolumn{2}{|l|}{$13.4 \pm 0.9$} & $13.9 \pm 1.4$ & $* *$ & $12.7 \pm 1.4$ \\
\hline & & & $* *$ & & \\
\hline \multirow[t]{2}{*}{ T-MNF } & \multirow[t]{2}{*}{$12.7 \pm 0.9$} & $*$ & $11.0 \pm 1.8$ & $* *$ & $8.5 \pm 1.3$ \\
\hline & & & $* *$ & & \\
\hline \multirow[t]{2}{*}{ MN-MF } & \multirow[t]{2}{*}{$25.6 \pm 1.2$} & $* *$ & $22.3 \pm 2.2$ & & $21.9 \pm 2.3$ \\
\hline & & & $*$ & & \\
\hline IB-MF & \multicolumn{2}{|l|}{$27.1 \pm 1.1$} & $26.5 \pm 1.9$ & $*$ & $25.0 \pm 2.1$ \\
\hline RB-MF & \multicolumn{2}{|l|}{$19.4 \pm 2.3$} & \multicolumn{2}{|l|}{$17.0 \pm 2.7$} & $16.9 \pm 2.3$ \\
\hline MC-IB & \multicolumn{2}{|l|}{$58.9 \pm 1.8$} & \multicolumn{2}{|l|}{$58.6 \pm 3.9$} & $59.7 \pm 4.1$ \\
\hline \multicolumn{6}{|l|}{ Mandibular body } \\
\hline IF-CF & \multicolumn{2}{|l|}{$15.1 \pm 1.2$} & $15.1 \pm 1.3$ & $*$ & $16.0 \pm 1.8$ \\
\hline BF-CF & \multicolumn{2}{|l|}{$2.6 \pm 0.7$} & \multicolumn{2}{|l|}{$2.1 \pm 1.2$} & $2.8 \pm 1.0$ \\
\hline IS-CS & \multicolumn{2}{|l|}{$17.0 \pm 1.9$} & $17.2 \pm 1.5$ & $*$ & $17.4 \pm 2.2$ \\
\hline BS-CS & \multicolumn{2}{|l|}{$3.6 \pm 0.7$} & $2.6 \pm 1.4$ & $*$ & $3.4 \pm 1.2$ \\
\hline
\end{tabular}

ramus osteotomy is to be performed, and to observe postoperative morphological change. It has been reported that CT measurement values had an error margin of within $3 \%$ when the images had a slice thickness of $2 \mathrm{~mm}$ and scanning was performed at $0.2-\mathrm{mm}$ intervals using non-contact, high-speed, 3D shape measurement equipment ${ }^{9}$. The present study used CT images with a slice width of $1.25 \mathrm{~mm}$, so the measurements obtained are believed not to involve a large error.

Horizontal osteotomy of the medial side in sagittal split ramus osteotomy involves adding as far as the mandibular ramus posterior bor- der above the mandibular foramen, and terminating at the posterior mandibular foramen above the mandibular foramen. There are two methods available for lateral osteotomy, with both involving addition as far as close to the mandibular angle and buccal cortical bone at a position below the molars ${ }^{24,25)}$. These procedures were designed to take into account the morphology and structure of the mandible. This is consistent with a report that mandibular thickness was reduced when a masticatory disturbance due to jaw deformity reduced mechanical stimulus $^{4)}$. To our knowledge, however, no study to 
Table 4 Multiple regression analysis

\begin{tabular}{|c|c|c|c|c|c|c|}
\hline $\begin{array}{c}\text { Dependent } \\
\text { variable }\end{array}$ & $\begin{array}{l}\text { Independent } \\
\text { variable }\end{array}$ & $\begin{array}{c}\text { Adjusted } \\
\text { R2 }\end{array}$ & F-value & $\begin{array}{l}\text { Regression } \\
\text { coefficient }\end{array}$ & $\begin{array}{l}\text { Standardized } \\
\text { regression } \\
\text { coefficient }\end{array}$ & $\mathrm{p}$-value \\
\hline \multicolumn{7}{|l|}{ Class I } \\
\hline MC-IB & SNB & 0.491 & 0.048 & -0.644 & -0.759 & 0.048 \\
\hline \multicolumn{7}{|l|}{ Class II } \\
\hline IB-MF & overjet & 0.122 & 0.026 & -0.59 & -0.386 & 0.026 \\
\hline RB-MF & $\begin{array}{c}\text { SNA } \\
\text { overjet }\end{array}$ & 0.185 & 0.018 & $\begin{array}{l}0.328 \\
0.749\end{array}$ & $\begin{array}{l}0.357 \\
0.335\end{array}$ & $\begin{array}{l}0.033 \\
0.044\end{array}$ \\
\hline MC-IB & overjet & 0.187 & 0.007 & -1.412 & -0.461 & 0.007 \\
\hline \multicolumn{7}{|l|}{ Class III } \\
\hline T-MNF & SNB & 0.117 & 0.011 & 0.172 & 0.369 & 0.011 \\
\hline MN-MF & Facial angle & 0.090 & 0.023 & -3.347 & -0.331 & 0.023 \\
\hline RB-MF & overbite & 0.100 & 0.017 & 0.597 & 0.346 & 0.017 \\
\hline MC-IB & $\begin{array}{c}\text { Y-axis } \\
\text { overbite }\end{array}$ & 0.135 & 0.016 & $\begin{array}{r}0.337 \\
-0.966\end{array}$ & $\begin{array}{r}0.342 \\
-0.281\end{array}$ & $\begin{array}{l}0.017 \\
0.048\end{array}$ \\
\hline BF-CF & $\begin{array}{c}\text { Y-axis } \\
\text { Facial angle }\end{array}$ & 0.263 & 0.000 & $\begin{array}{l}9.516 \\
1.776\end{array}$ & $\begin{array}{l}0.413 \\
0.370\end{array}$ & $\begin{array}{l}0.002 \\
0.005\end{array}$ \\
\hline BS-CS & $\begin{array}{c}\text { Y-axis } \\
\text { Facial angle }\end{array}$ & 0.250 & 0.001 & $\begin{array}{l}0.101 \\
2.099\end{array}$ & $\begin{array}{l}0.384 \\
0.383\end{array}$ & $\begin{array}{l}0.004 \\
0.004\end{array}$ \\
\hline
\end{tabular}

date has compared mandibular morphology among different facial skeletal types.

\section{Correlation between mandibular ramus morphology and facial skeletal morphology}

In Class I, the height of the mandibular ramus showed a decrease the more anterior the location of the mandibular alveolar base. In Class II, the larger the horizontal tegmentum, the smaller the distance of the mandibular foramen from the mandibular inferior border, and the lesser the height of the mandibular ramus. The more anterior the maxillary alveolar base, and the larger the horizontal tegmentum, the greater the distance of the mandibular foramen from the anterior border of the mandibular ramus. This implied that there was a tendency for a larger horizontal tegmentum and more anterior location of the mandibular alveolar base to mean a greater relative height of the position of the mandibular foramen to the height of the mandibular ramus.

In Class III, the more anterior the mandibular alveolar base, the greater the thickness of the mandibular ramus. Also, greater anteroinferior growth of the mandible, and a more anterior location of the chin, meant an increase in the distance of the mandibular canal from the buccal cortical bone in the mandibular body. It was anticipated that a more anterior position of the mandible would reduce the buccolingual thickness of the mandibular ramus, but the results showed that the thickness of the mandibular ramus was in fact greater, contrary to expectations. This suggests that, when a mandible shows prognathism, the mandibular ramus grows not only in the anteroinferior direction, but also in the buccolingual direction.

With respect to the position of the mandibular foramen, the more anterior the mandible, the smaller the distance between the mandibular notch and the mandibular foramen; and the greater the vertical tegmentum, 
the greater the distance of the mandibular foramen from the anterior border of the mandibular ramus. Greater anteroinferior growth of the mandible, and a smaller vertical tegmentum, meant an increase in the height of the mandibular ramus. The more anterior the mandible, the higher the position of the mandibular foramen relative to the height of the mandibular ramus, a finding that was also contrary to expectations. Mandibular growth is particularly active during endochondral bone formation in the mandibular condyle ${ }^{6,10)}$. The results of the present study suggest that the mandible in patients with jaw deformity exhibits active growth not only during formation of endochondral bone in the mandibular condyle, but also during formation of intramembranous bone.

\section{Consideration of ideal osteotomy sites}

In Class II, the distance of the mandibular canal from the buccal cortical bone showed no difference in comparison with other skeletal types in the area of the second molar. It was located more buccally, however, in the area of the first molar. This suggests that, when one is performing lateral osteotomy or sagittal splitting, there is a greater risk of nerve damage. If firm osteosynthesis is considered possible, then lateral osteotomy is believed to reduce risk of damage to the mandibular canal in comparison with posterior osteotomy. With medial osteotomy, there were no indicators for selection of osteotomy type, as there were no features related to skeletal type.

In Class III, there was a marked tendency for the thickness of the mandibular ramus to decrease, moving upward from the mandibular foramen. The relative position of the mandibular foramen in the mandibular ramus was also higher than that in the other skeletal types. In addition, the more anterior the mandibular alveolar base, the greater the thickness of the mandibular ramus. With a short lingual cut method, there are some instances where trimming of the posterior mandibular ramus is necessary after sagittal splitting. If the mandibular ramus is very thick, we believe that performing the osteotomy as far as the mandibular ramus posterior border will obviate the need for trimming and simplify the procedure. With lateral osteotomy, however, there are no features peculiar to a particular facial skeletal type, which means it is impossible to identify potential indicators for selection of osteotomy method.

\section{Acknowledgements}

The authors would like to thank Professor Jeremy Williams, Tokyo Medical University, for his assistance with the English of this manuscript.

\section{References}

1) Baas EM, Pijpe J, de Lange J (2012) Long term stability of mandibular advancement procedures: bilateral sagittal split osteotomy versus distraction osteogenesis. Int J Oral Maxillofac Surg 41:137-141.

2) Cillo JE, Stella JP (2005) Selection of sagittal split ramus osteotomy technique based on skeletal anatomy and planned distal segment movement: Current therapy. J Oral Maxillofac Surg 63:109-114.

3) Fong JH, Wu HT, Huang MC, Chou YW, Chi LY, Fong Y, Kao SY (2010) Analysis of facial skeletal characteristics in patients with chin deviation. J Chin Med Assoc 73:29-34.

4) Hayashi M, Isshiki Y, Nishikawa K (2001) Morphological and quantitative analyses on features of asymmetric mandible with X-ray CT images. Shikwa Gakuho 101:835-848.

5) Kim $\mathrm{CH}$, Lee JH, Cho JY, Lee JH, Kim KW (2007) Skeletal stability after simultaneous mandibular angle resection and sagittal split ramus osteotomy for correction of mandible prognathism. J Oral Maxillofac Surg 65: 192-197.

6) Kim S, Motegi E, Kikuchi Y, Yamaguchi H, Takaki T, Shibahara T (2007) Possibility of estimating three-dimensional mandibular morphology by cephalogram analysis. Shikwa Gakuho 107:323-330.

7) Komori E, Aigase K, Sugisaki M, Tanabe H (1989) Cause of early skeletal relapse after mandibular setback. Am J Orthod Dentofacial Orthop 95:29-36. 
8) Levine MH, Goddard AL, Dodson TB (2007) Inferior alveolar nerve canal position: A clinical and radiographic study. J Oral Maxillofac Surg 65:470-474.

9) Matsumoto K, Maki K, Nakano H, Shibasaki Y (2000) A study on the calculation of root surface area and the evaluation of anchorage value employing CT images. Dental Med Res 20:62-68.

10) Mizoguchi I (1999) Growth behavior of the mandible and biological aspects of mandibular condaylar cartilage. Tohoku Univ Dent J 18:1-21.

11) MoriY, Shimizu H, Minami K, Kwon TG, Mano T (2011) Development of a simulation system in mandibular orthognathic surgery based on integrated three-dimensional data. J Oral Maxillofac Surg 15:131-138.

12) Naruse T (1998) EMG topographic analysis of facial-muscle activities in patients with maxillary protrusion and mandibular anterior protrusion. Shikwa Gakuho 98:661-683.

13) Park KR, Kim SY, Kim GJ, Park HS, Jung YS (2014) Anatomic study to determine a safe surgical reference point for mandibular ramus osteotomy. J Cranio Maxillofac Surg 42:22-27.

14) Precious DS (2004) Removal of third molars with sagittal split osteotomies. J Oral Maxillofac Surg 62:1144-1146.

15) Rothman SL, Chaftez N, Rhodes ML, Schwarz MS (1988). CT in the preoperative assessment of the mandible and maxilla for endosseous implant surgery. Radiology 168:171-175.

16) Shimada J, Furuya A, Takeshima H, Nagamine K, Okitsu M, Yamamoto Y (1990) Anatomical and morphological study of mandibular ramus relating to sagittal splitting osteotomy. Jpn J Oral Maxillofac Surg 36:24-36.

17) Smith BR, Rajchel JL, Waite DE, Read L (1991) Mandibular ramus anatomy as it relates to the medial osteotomy of the sagittal split ramus osteotomy. J Oral Maxillofac Surg 49: 112-116.

18) Smith GI, Brennan PA, Oh SS, Markus AF (2002) Modification of the Hunsuck sagittal split osteotomy using a nerve hook. J Cranio Maxillofac Surg 30:292-294.

19) Song JM, Kim YD (2014) Three-dimensional evaluation of lingual split line after bilateral sagittal split osteotomy in asymmetric prognathism. J Korean Assoc Oral Maxillofac Surg 40: 11-16.

20) Trauner R, Obwegeser H (1957) The surgical correction of mandibular prognathism and retrognathia with consideration of genioplasty. Oral Surg Oral Med Oral Pathol 10: 677-689.

21) Tsuji Y, Muto T, Kawakami J, Takeda S (2005) Computed tomographic analysis of the position and course of the mandibular canal: relevance to the sagittal split ramus osteotomy. Int J Oral Maxillofac Surg 34:243-246.

22) Turvey TA (1985) Intraoperative complications of sagittal osteotomy of the mandibular ramus: incidence and management. J Oral Maxillofac Surg 43:504-509.

23) Ueki K, Okabe K, Miyazaki M, Mukozawa A, Marukawa K, Nakagawa K, Yamamoto E (2010) Position of mandibular canal and ramus morphology before and after sagittal split ramus osteotomy. J Oral Maxillofac Surg 68:1795-1801.

24) Wolford LM, Bennett MA, Rafferty CG (1987) Modification of the mandibular ramus sagittal split osteotomy. Oral Surg Oral Med Oral Pathol 64:146-155.

25) Wolford LM (2000) The sagittal split ramus osteotomy as the preferred treatment for mandibular prognathism. J Oral Maxillofac Surg 58:310-312.

Correspondence:

Dr. Takahiko Shibahara

Department of Oral \& Maxillofacial

Surgery,

Tokyo Dental College,

2-9-18 Kanda-Misakicho, Chiyoda-ku,

Tokyo 101-0061, Japan

E-mail: sibahara@tdc.ac.jp 